\title{
Factorization and Non-Factorization of In-Medium Four-Quark Condensates
}

\author{
Stefan Leupold \\ Institut für Theoretische Physik, Universität Giessen, Germany
}

\begin{abstract}
It is well-established for the vacuum case that in the limit of a large number of colors $N_{c}$ the four-quark condensates factorize into products of the two-quark condensate. It is shown that in the combined large- $N_{c}$ and linear-density approximation four-quark condensates do not factorize in a medium of pions (finite temperature system) but do factorize in a medium of nucleons (nuclear system).
\end{abstract}

PACS numbers: 12.38.Lg,11.15.Pg,21.65.+f,11.10.Wx

Keywords: QCD sum rules, large-Nc expansion, nuclear matter, finite temperature field theory

It is by now well accepted that QCD, the theory of the strong interaction, has a non-trivial vacuum structure. One way to characterize this structure is by means of non-vanishing matrix elements of quark or gluon operators, the condensates. Particular condensates also play an important role for the connection of hadronic properties to the underlying quark-gluon structure, as investigated in much detail within the QCD sum rule method [1, 2]. Most prominently, the two-quark condensate, the gluon condensate and four-quark condensates appear in the sum rules. When it comes to numbers the largest uncertainties reside in the actual values for the four-quark condensates. They remained a matter of constant debate over the last decades [1, 3, 4, 5, 6, 7, 8, [9]. One central point in these discussions is the question whether a four-quark condensate can be factorized more or less accurately into a product of two-quark condensates.

In hot and/or dense enough strongly interacting media, QCD is subject to phase transitions or rapid crossovers 10, 11]. Consequently, the condensates typically change with density and temperature. In lowest non-trivial order in temperature and density the changes of the two-quark and the gluon condensate are well established 12, 13, 14, 15]. The issue is much less clear for the four-quark condensates [8, 9, 14, 16, 17, 18]. To summarize, for the vacuum values as well as for the in-medium changes the uncertainties are the largest for the four-quark condensates.

There is one particular limit of QCD, however, where exact relations can be obtained, namely the limit of a large number of colors $N_{c}$. While the physical $\left(N_{c}=3\right)$ values of four-quark condensates are a matter of debate, at least in the large- $N_{c}$ limit the factorization into two-quark condensates holds for the vacuum case [19]. In the present work we generalize this aspect to typical in-medium cases. We will show that in the large- $N_{c}$ limit an arbitrary four-quark condensate factorizes in a nuclear medium, while it does not in a pionic medium. The pion case has already been discussed in detail in 14, 16] from a different point of view. The nucleon case was addressed only recently by the present author 20]. In the following, we present a unified derivation for pions and nucleons.

We start by recalling how some hadronic properties scale as a function of the number of colors $N_{c}$. According to [21, 22] the following rules hold for the expansion of hadronic quantities in powers of $1 / N_{c}$ :

- Mesons are $q \bar{q}$-states, their masses are $O\left(N_{c}^{0}\right)$.

- In contrast, a baryon consists of $N_{c}$ quarks, its mass is $O\left(N_{c}\right)$.

- The ratio between a quark current $A$ and the corresponding interpolating hadronic field $A_{H}$ is $O\left(\sqrt{N_{c}}\right)$.

- Mesonic interactions are suppressed. Generically $n$-meson vertices are only $O\left(N_{c}^{1-n / 2}\right)$. Consequently, the decay width of a meson into two other mesons is $O\left(1 / N_{c}\right)$. Therefore, in vacuum mesons are stable in the large- $N_{c}$ limit.

- In contrast, meson-baryon interactions are not suppressed. Meson-baryon scattering amplitudes are $O\left(N_{c}^{0}\right)$. A meson-baryon-baryon vertex can even be enhanced as $O\left(\sqrt{N_{c}}\right)$. (Subtle cancellations ensure that these two rules are compatible, cf. e.g. [23].)

Using Fierz transformations, in particular 24]

$$
\frac{1}{2}\left(\lambda_{a}\right)_{\alpha \beta}\left(\lambda_{a}\right)_{\gamma \delta}=\delta_{\alpha \delta} \delta_{\beta \gamma}-\frac{1}{N_{c}} \delta_{\alpha \beta} \delta_{\gamma \delta},
$$

every four-quark operator

$$
\bar{\psi} \lambda_{a} \Gamma \psi \bar{\psi} \lambda_{a} \Gamma^{\prime} \psi
$$


can be written as a sum of products of two color neutral two-quark operators

$$
\bar{\psi} \Gamma^{\prime \prime} \psi
$$

Here $\lambda_{a}$ denotes a color matrix and $\Gamma, \Gamma^{\prime}, \Gamma^{\prime \prime}$ matrices in spinor and flavor space. Therefore we can restrict our

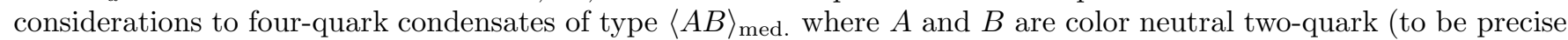
quark-antiquark) operators of type (33). In the following we will interpret $A$ and $B$ as interpolating fields for hadrons. Effectively we will assume that color is saturated within $A$ and $B$ separately. In other words: The quark and the antiquark field of $A$ form a hadron, same for $B$. In that way, however, we neglect possible exchange terms, i.e. the ones where e.g. the quark field from $A$ and the antiquark field from $B$ form a hadron. Fortunately these exchange terms are subleading in the number of colors and therefore of no concern for our present considerations. This can be seen as follows:

$$
\overline{\bar{q}_{i} \Gamma q_{i}} \overline{\bar{q}_{j} \Gamma^{\prime} q_{j}} \rightarrow \delta_{i i} \delta_{j j}=N_{c}^{2}
$$

whereas

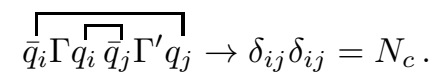

Here the connection... indicates that the two respective fields form hadronic states.

We approximate the medium by a gas of non-interacting states of type $X$ with density $\rho_{X}$. This is a valid approximation as long as the density is not too high. The extension to a gas composed of several species is straightforward. For a system at finite temperature (nuclear density) one uses pions (nucleons) for $X$. We get

$$
\langle A B\rangle_{\text {med. }} \approx\langle 0|A B| 0\rangle+\rho_{X}\langle X|A B| X\rangle
$$

where $|0\rangle$ denotes the vacuum state. The expectation value with respect to the single state $X$ consists of five parts: First, the transition process $A+X$ to $X$, with $B$ as a pure spectator; second, the same process with the roles of $A$ and $B$ reversed; third, the annihilation of $X$ by $B$ and the creation of $X$ by $A$; fourth, the latter process with the roles of $A$ and $B$ reversed; finally, the true scattering of $B$ with $X$ into $A$ and $X$. Denoting the true scattering process by $\langle X|A B| X\rangle_{\text {connected }}$ we get the following decomposition:

$$
\langle X|A B| X\rangle=\langle X|A| X\rangle\langle 0|B| 0\rangle+\langle 0|A| 0\rangle\langle X|B| X\rangle+\langle X|A| 0\rangle\langle 0|B| X\rangle+\langle 0|A| X\rangle\langle X|B| 0\rangle+\langle X|A B| X\rangle_{\text {connected }} .
$$

So far, everything was rather general. In particular, we have not involved any large- $N_{c}$ arguments besides the dropping of the exchange terms. Now we recall that a quark current $A$ is connected to mesonic fields $A_{H}$ with the same quantum numbers by a factor which is $O\left(\sqrt{N_{c}}\right)$. Hence the connected part in (7) is of order $N_{c}$ times a hadronic scattering amplitude. The size of the latter depends on $X$, but it is at most $O\left(N_{c}^{0}\right)$. Below we will find that this term is always subleading. Independent of $X$ we can already factorize the vacuum part in (6) in the large- $N_{c}$ approximation:

$$
\begin{aligned}
\langle A B\rangle_{\text {med. }} \approx & \langle 0|A| 0\rangle\langle 0|B| 0\rangle+o\left(N_{c}\right) \\
& +\rho_{X}\langle X|A| X\rangle\langle 0|B| 0\rangle+\rho_{X}\langle 0|A| 0\rangle\langle X|B| X\rangle \\
& +\rho_{X}\langle X|A| 0\rangle\langle 0|B| X\rangle+\rho_{X}\langle 0|A| X\rangle\langle X|B| 0\rangle+\rho_{X}\langle X|A B| X\rangle_{\text {connected }}
\end{aligned}
$$

Note that the leading part here is $O\left(N_{c}^{2}\right)$ since $\langle 0|A| 0\rangle=O\left(N_{c}\right)$.

On the right hand side of (8) the terms given explicitly in the first two lines constitute the result of a factorization assumption:

$$
\begin{gathered}
\langle A\rangle_{\text {med. }}\langle B\rangle_{\text {med. }}=\left(\langle 0|A| 0\rangle+\rho_{X}\langle X|A| X\rangle\right)\left(\langle 0|B| 0\rangle+\rho_{X}\langle X|B| X\rangle\right)= \\
\langle 0|A| 0\rangle\langle 0|B| 0\rangle+\rho_{X}\langle X|A| X\rangle\langle 0|B| 0\rangle+\rho_{X}\langle 0|A| 0\rangle\langle X|B| X\rangle+o\left(\rho_{X}^{2}\right) .
\end{gathered}
$$

(Note that we disregard terms quadratic in the density as we work in the linear-density approximation.) On the other hand, the terms given in the last line of (8) in general spoil the factorization assumption. In the following we will find that in leading order of a $1 / N_{c}$ expansion these terms are present for the case of finite temperatures but absent for the case of finite baryon densities.

To get a first glance how the large- $N_{c}$ rules work we shall prove the vacuum factorization: We start by inserting a (hadronic) unity operator between $A$ and $B$ and get

$$
\langle 0|A B| 0\rangle=\langle 0|A| 0\rangle\langle 0|B| 0\rangle+\sum_{h}\langle 0|A| h\rangle\langle h|B| 0\rangle+\text { insertions of multi-particle states. }
$$


As already stated the first term on the right hand side is $O\left(N_{c}^{2}\right)$. The next term is only $O\left(N_{c}\right)$ due to

$$
\langle 0|A| h\rangle \sim \sqrt{N_{c}}\left\langle 0\left|A_{H}\right| h\right\rangle \sim \sqrt{N_{c}} .
$$

The multi-particle states are even further suppressed. Hence in the vacuum any four-quark condensate factorizes in leading order of the $1 / N_{c}$ expansion.

To make further progress we have to specify $X$. On account of the rules discussed above we have for pions

$$
\begin{aligned}
& \langle\pi|A| \pi\rangle \sim \sqrt{N_{c}}\left\langle\pi\left|A_{H}\right| \pi\right\rangle \sim N_{c}^{0}, \\
& \langle\pi|A| 0\rangle \sim \sqrt{N_{c}}\left\langle\pi\left|A_{H}\right| 0\right\rangle \sim \sqrt{N_{c}}
\end{aligned}
$$

and

$$
\langle\pi|A B| \pi\rangle_{\text {connected }} \sim N_{c}\left\langle\pi\left|A_{H} B_{H}\right| \pi\right\rangle_{\text {connected }} \sim N_{c}^{0},
$$

whereas for nucleons we find

$$
\begin{gathered}
\langle N|A| N\rangle \sim \sqrt{N_{c}}\left\langle N\left|A_{H}\right| N\right\rangle \sim N_{c}, \\
\langle N|A| 0\rangle=0
\end{gathered}
$$

and

$$
\langle N|A B| N\rangle_{\text {connected }} \sim N_{c}\left\langle N\left|A_{H} B_{H}\right| N\right\rangle_{\text {connected }} \sim N_{c} .
$$

Of course, all relations displayed for $A$ hold also for $B$. Note that $A$ and $B$ are quark-antiquark operators. Hence they cannot create a nucleon out of the vacuum. Therefore $\langle N|A| 0\rangle$ has to vanish as stated in (16). This will constitute the important difference which makes the temperature and the nuclear density case distinct from each other.

We conclude, first of all, that all pionic in-medium effects are suppressed as compared to the vacuum part:

$$
\langle A B\rangle_{\text {pionic med. }} \approx\langle 0|A| 0\rangle\langle 0|B| 0\rangle+o\left(N_{c}\right) .
$$

If we keep the highest non-trivial order in $N_{c}$ of the medium part, we get

$$
\begin{aligned}
& \langle A B\rangle_{\text {pionic med. }} \approx\langle 0|A| 0\rangle\langle 0|B| 0\rangle+o\left(N_{c}\right) \\
& \quad+\rho_{\pi}\left(\langle\pi|A| \pi\rangle\langle 0|B| 0\rangle+\langle 0|A| 0\rangle\langle\pi|B| \pi\rangle+\langle\pi|A| 0\rangle\langle 0|B| \pi\rangle+\langle 0|A| \pi\rangle\langle\pi|B| 0\rangle+o\left(N_{c}^{0}\right)\right) .
\end{aligned}
$$

Obviously even now factorization is spoiled by the annihilation and creation terms like $\langle\pi|A| 0\rangle\langle 0|B| \pi\rangle$. Such terms do not vanish, if $A$ and $B$ have the quantum numbers of the pion. ${ }^{1}$

In contrast, in a nuclear medium we find "factorization":

$$
\langle A B\rangle_{\text {nuclear med. }} \approx\langle 0|A| 0\rangle\langle 0|B| 0\rangle+\rho_{N}\langle N|A| N\rangle\langle 0|B| 0\rangle+\rho_{N}\langle 0|A| 0\rangle\langle N|B| N\rangle+o\left(N_{c}\right) .
$$

The quotation marks are meant to indicate that there is no $\rho_{N}^{2}$ term which would appear if factorization was taken literally.

To summarize, we have addressed a limit in which for the vacuum case the four-quark condensates can be expressed in terms of the two-quark condensate, namely the large- $N_{c}$ limit. We have shown that in the same limit we can make exact statements for in-medium expectation values of four-quark condensates. In particular, reproducing the results of 14, 16] with a different technique we have found that in the commonly used linear-density approximation in general four-quark condensates do not factorize for the finite-temperature case. In addition, we have found that (also in linear-density approximation) for the nuclear medium four-quark condensates factorize into two-quark condensates. This qualitative difference has a simple reason: Among the terms which potentially spoil the factorization (last line in

\footnotetext{
${ }^{1}$ Note that the matrix elements which appear in [19] can be calculated using current algebra [14, 16].
} 
(8) ) the true scattering process is always subleading in $1 / N_{c}$ as compared to the in-medium terms which show up in a factorization assumption (cf. (9)). The creation and annihilation terms, on the other hand, — if present — contribute with the same power in $1 / N_{c}$ as the terms in (9). In a mesonic medium these annihilation and creation terms can be non-vanishing whereas in a baryonic medium they vanish.

Finally, we note that with the decomposition (7) and the interpretation of $\langle X|A B| X\rangle_{\text {connected as a true scattering }}$ amplitude it might be feasible to estimate this amplitude from hadronic models. (This is similar in spirit to the approach presented in [25].) Especially the experimentally well explored pion-nucleon scattering amplitude can be used. First results in that direction are rather satisfying as they indicate that corrections to (20) are quite small. A more detailed investigation is now in progress. Besides phenomenology again the large- $N_{c}$ expansion might be helpful: For a given hadronic model (e.g. with the well-known physical states $\pi, \rho, \omega, N$ and $\Delta$ ) one can calculate meson-nucleon scattering in the large- $N_{c}$ limit to all loop orders [26, 27]. This would yield the next-to-leading $O\left(N_{c}\right)$ term in (20). In that way the validity of the in-medium factorization (20) could be checked beyond the leading order of the $1 / N_{c}$ expansion.

\section{Acknowledgments}

The author thanks A. Peshier for discussions and for reading the manuscript and U. Mosel for continuous support.

[1] M. A. Shifman, A. I. Vainshtein, and V. I. Zakharov, Nucl. Phys. B147, 385, 448 (1979).

[2] L. J. Reinders, H. Rubinstein, and S. Yazaki, Phys. Rept. 127, 1 (1985).

[3] G. Launer, S. Narison, and R. Tarrach, Z. Phys. C26, 433 (1984).

[4] R. A. Bertlmann, C. A. Dominguez, M. Loewe, M. Perrottet, and E. de Rafael, Z. Phys. C39, 231 (1988).

[5] C. A. Dominguez and J. Sola, Z. Phys. C40, 63 (1988).

[6] V. Gimenez, J. Bordes, and J. Penarrocha, Nucl. Phys. B357, 3 (1991).

[7] D. B. Leinweber, Annals Phys. 254, 328 (1997), nucl-th/9510051.

[8] F. Klingl, N. Kaiser, and W. Weise, Nucl. Phys. A624, 527 (1997), hep-ph/9704398.

[9] S. Leupold, W. Peters, and U. Mosel, Nucl. Phys. A628, 311 (1998), nucl-th/9708016.

[10] F. Karsch, Lect. Notes Phys. 583, 209 (2002), hep-lat/0106019.

[11] K. Rajagopal and F. Wilczek, in At the Frontier of Particle Physics: Handbook of QCD: Boris Ioffe Festschrift, edited by M. Shifman (World Scientific, Singapore, 2002), vol. 3, pp. 2061-2151, hep-ph/0011333.

[12] P. Gerber and H. Leutwyler, Nucl. Phys. B321, 387 (1989).

[13] E. G. Drukarev and E. M. Levin, Prog. Part. Nucl. Phys. 27, 77 (1991).

[14] T. Hatsuda, Y. Koike, and S. H. Lee, Nucl. Phys. B394, 221 (1993).

[15] T. Hatsuda and S. H. Lee, Phys. Rev. C46, 34 (1992).

[16] V. L. Eletsky, Phys. Lett. B299, 111 (1993).

[17] S. Zschocke, O. P. Pavlenko, and B. Kämpfer, Phys. Lett. B562, 57 (2003), hep-ph/0212201.

[18] S. Zschocke, O. P. Pavlenko, and B. Kämpfer (2003), hep-ph/0308070.

[19] V. A. Novikov, M. A. Shifman, A. I. Vainshtein, M. B. Voloshin, and V. I. Zakharov, Nucl. Phys. B237, 525 (1984).

[20] S. Leupold and M. Post, Nucl. Phys. A747, 425 (2005), nucl-th/0402048.

[21] G. 't Hooft, Nucl. Phys. B72, 461 (1974).

[22] E. Witten, Nucl. Phys. B160, 57 (1979).

[23] C. S. Lam and K. F. Liu, Phys. Rev. Lett. 79, 597 (1997), hep-ph/9704235.

[24] P. Pascual and R. Tarrach, QCD: Renormalization for the Practitioner, vol. 194 of Lect. Notes Phys. (Springer, Berlin, 1984).

[25] V. L. Eletsky, P. J. Ellis, and J. I. Kapusta, Phys. Rev. D47, 4084 (1993), hep-ph/9302300.

[26] P. B. Arnold and M. P. Mattis, Phys. Rev. Lett. 65, 831 (1990).

[27] M. P. Mattis and R. R. Silbar, Phys. Rev. D51, 3267 (1995), hep-ph/9405366. 